\title{
L'origine des métazoaires
}

\section{Herman Denis, Jean-Piere Mignot}

On pense que les métazoaires dérivent d'organismes coloniaux, qui dérivent eux-mêmes de protozoaires. La formation des métazoaires requit de nombreuses innovations. Il fallait faire adhérer et communiquer ensemble des cellules, puis leur permettre de se spécialiser. Il fallait aussi édifier à chaque génération un nouvel organisme à partir d'une seule cellule, donc inventer une embryogenèse. Les choses furent certainement facilitées par les progrès déjà réalisés en matière de reproduction par les prédécesseurs des métazoaires.

Chez de nombreux protozoaires et certains organismes coloniaux, le cycle vital fait alterner une phase végétative avec une phase sexuée. Durant la phase végétative, les cellules se multiplient par mitose. Pendant la phase sexuée, les cellules se convertissent en gamètes mâles et femelles, qui fusionnent en un zygote. Deux programmes génétiques distincts gouvernent la phase végétative et la phase sexuée. Ils sont exécutés l'un après l'autre par des cellules de même lignée. Les métazoaires ont conservé ces deux programmes, mais les font simultanément exécuter par des cellules d'origine embryologique différente. Les cellules somatiques mettent en cuvre le programme végétatif. Les cellules germinales réalisent le programme sexué. L'embryogenèse la plus simple consiste en une dichotomie. Elle n'engendre que deux catégories de cellules, somatiques et germinales. C'est probablement de cette facon que s'organisaient les premiers métazoaires. $\mathrm{m} / \mathrm{s} n^{\circ} 5$ vol. 10, mai 94

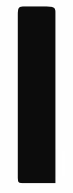
l y a plus d'un siècle que les biologistes s'intéressent à l'origine des métazoaires [1-3]. Ils se posent deux questions essentielles: quand et comment les animaux multicellulaires sont-ils apparus? La paléontologie fournit des éléments de réponse. Mais elle nous livre un message plutôt décourageant. Il est impossible de savoir comment les premiers métazoaires étaient construits, car les fossiles les plus anciens attestent une assez longue évolution antérieure. Il s'agit de restes d'animaux déjà diversifiés, présentant plusieurs modes d'organisation. Ces vestiges appartiennent à la faune dite d'Ediacara, exhumée d'abord en Australie, puis dans d'autres continents. Leur enfouisse ment remonte à 600 millions d'années et même davantage, bien avant le début de l'ère primaire (paléozoïque) [3, 4].

\section{Principes de phylogenèse}

Faute d'observations paléontologiques suffisamment anciennes et précises, on est réduit à des conjectures. On doit comparer les différents métazoaires pour essayer d'imaginer à quoi ressemblaient les premiers d'entre eux. Haeckel fut le premier à tenter cet exercice [1]. Inspiré par les idées de Lamarck et de Darwin, Haeckel proposa une phylogénie globale, qu'il matérialisa par un arbre. Cette figure retrace l'histoire évolutive des métazoaires. Les extrémités des rameaux symbolisent les groupes zoologiques actuels. Les branches représentent les diverses lignées animales. Chaque ramification évoque un événement passé, imaginé par l'auteur: une lignée ancestrale se divise en deux lignées filles, qui divergent progressivement. Comme dans un arbre réel, les bifurcations les plus proches du pied correspondent aux dichotomies les plus anciennes. Le tronc émerge d'un substrat formé par des organismes unicellulaires.

En un siècle, on a proposé beaucoup d'arbres phylogénétiques, également appelés cladogrammes. Jusqu'au milieu des années 1960, la construction des cladogrammes reposait sur des critères morphologiques. Par la suite, on vit apparaître des phylogénies moléculaires, fondées sur des comparaisons entre les séquences d'un seul gène, ou du produit d'un seul gène (ARN ou protéine). Quelle que soit la méthode employée, la construction des arbres s'appuie sur quelques principes simples, utilisés par tous les auteurs de façon explicite ou implicite $[5,6]$. Ces principes peuvent être formulés comme suit.

1. Les groupes d'organismes les plus semblables occupent les positions les plus proches dans l'arbre phylogénétique.

2. La ressemblance entre les groupes se mesure en dénombrant les caractères identiques ou similaires qu'ils partagent.

3. Il ne faut tenir compte que des similitudes reflétant une communauté d'origine. On appelle homologues les caractères semblables héri- 
tés d'un ancêtre commun, ainsi que les gènes qui spécifient ces caractères. On appelle convergents les caractères semblables apparus dans des lignées différentes, à la suite de processus évolutifs indépendants (figure 1).

4. Les caractères homologues se partagent en caractères ancestraux et caractères dérivés. Les caractères ancestraux préexistent à une dichotomie. Ils se retrouvent dans les lignées filles (figure 1). Les caractères dérivés sont d'apparition plus tardive. Ils sont présents dans une branche déterminée et les rameaux qui en émanent, mais absents dans les branches issues de bifurcations antérieures (figure 1).

5. Pour relier les groupes entre eux il faut procéder avec économie, en postulant le moins possible de transformations évolutives. Si plusieurs arbres phylogénétiques sont compatibles avec une étude comparative de caractères, on tiendra l'arbre le plus simple pour le plus vraisemblable. Des divergences apparaissent dans les arbres phylogénétiques élaborés par les divers auteurs. Les variations observées concernent notamment l'origine des métazoaires. Lorsque les cladogrammes ont une base morphologique, les difficultés résident dans l'application du concept d'homologie. Il est parfois malaisé, voire impossible, de décider si deux caractères structuraux sont homologues ou convergents. Quand il s'agit d'acides nucléiques ou de protéines, la décision semble plus facile à prendre. On se fonde alors sur des critères statistiques. Sont jugés homologues les polymères dont les séquences se ressemblent. Beaucoup d'ambiguïtés seraient levées si l'on adoptait comme critères décisifs d'homologie des similitudes de séquence. On devrait considérer comme homologues les structures (organites, organes, appendices) dont l'édification est gouvernée par un ensemble de gènes apparentés. Les mêmes critères peuvent s'appliquer aux mécanismes biochimiques.

Les phylogénies moléculaires semblent échapper au problème qui vient d'être évoqué, puisqu'elles s'appuient sur des comparaisons homologues. Pourtant, les divers arbres proposés révèlent de nombreuses discordances [7-10], dont la cause n'est pas encore clairement établie.

\section{Monophylétisme ou polyphylétisme}

Concernant l'origine des métazoaires, un point fait l'objet d'un large assentiment: les ancêtres directs des animaux sont à chercher parmi les protozoaires [1, 6, 11-15]. Mais un problème crucial fait encore l'objet de débats: les métazoaires sont-ils apparus à une seule reprise ou à plusieurs reprises au cours de l'évolution? La thèse monophylétique a ses partisans $[2,3,12,15]$. La thèse polyphylétique a également les siens $[6,11,13]$. La même incertitude ressort de l'examen des phylogénies moléculaires fondées sur des comparaisons entre séquences d'ARN ribosomique [7-10].

Il est probable qu'une réponse claire émergera tôt ou tard des analyses moléculaires. En attendant, on peut essayer de prendre parti en utilisant les principes de phylogenèse énoncés plus haut. Il s'agit de

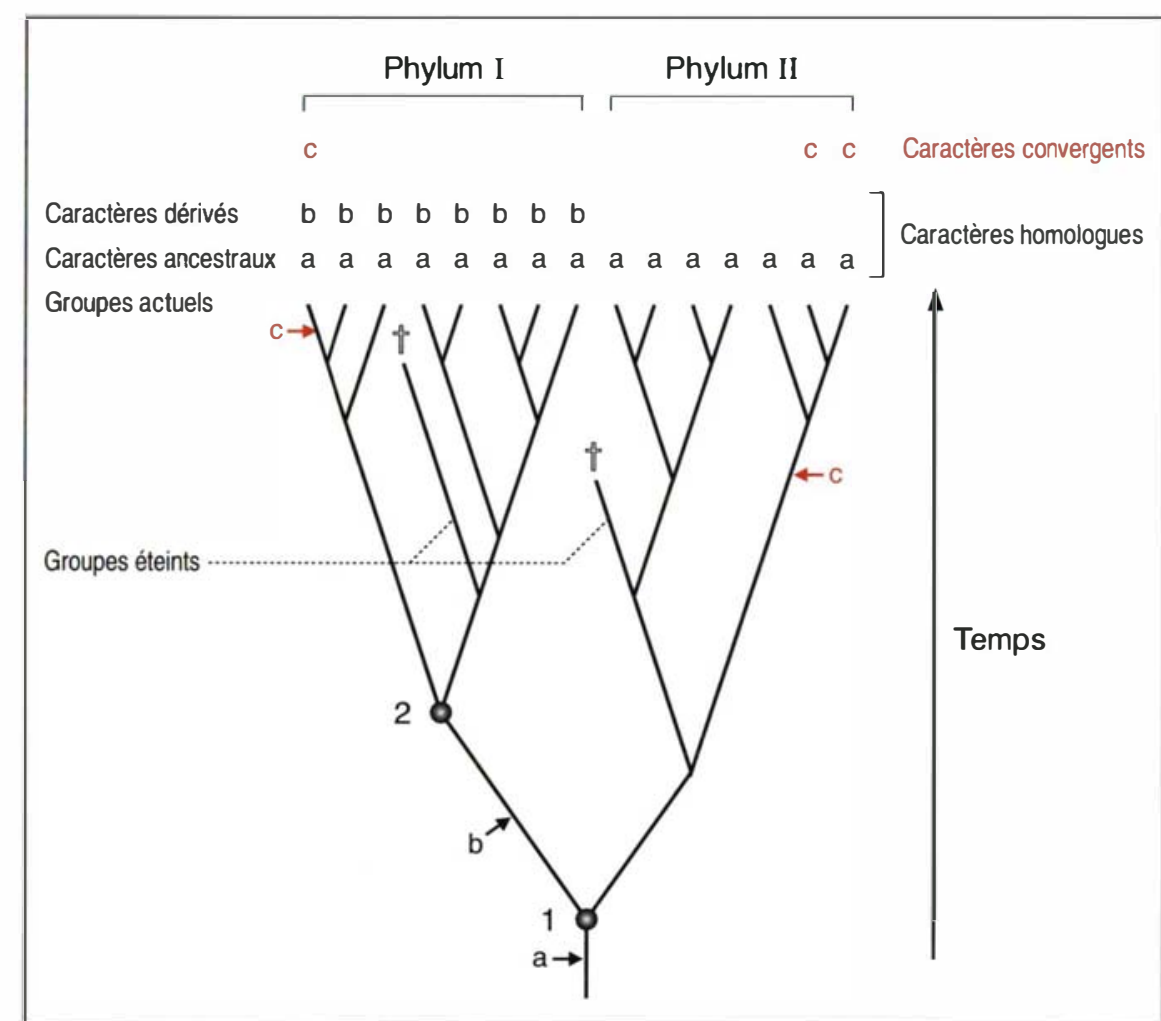

Figure 1. Un arbre phylogénétique idéal. Le schéma retrace l'histoire évolutive d'un ensemble d'organismes apparentés, répartis en deux phylums. Tous ces organismes sont censés dériver d'un même ancêtre. Le tronc, les branches et les rameaux représentent diverses lignées d'organismes anciens, dont chacune aboutit à un groupe actuel ou éteint. Les ramifications connectent les différents groupes en fonction du nombre de caractères identiques ou similaires qu'ils partagent. Pour établir les filiations, il faut négliger les caractères convergents et ne prendre en compte que les caractères homologues. Sont considérés comme convergents (tels que c) des caractères semblables apparus indépendamment dans plusieurs lignées. Sont considérés comme homologues (tels que a et b) des caractères apparus dans une lignée parentale et conservés par toutes ou certaines lignées filles. Parmi les caractères homologues, on distingue ceux qui sont ancestraux de ceux qui sont dérivés. Par exemple, le caractère a est ancestral. On le trouve dans les phylums I et II. Son apparition est donc antérieure à la première dichotomie. Le caractère b est dérivé par rapport au caractère a. II n'existe que dans le phylum I. Son apparition se situe entre les dichotomies 1 et 2. 
rechercher si tous les métazoaires possèdent des caractères dérivés, donc absents chez les autres organismes multicellulaires et chez les protistes. Si c'est le cas, l'hypothèse monophylétique sera confortée. Nous énumérons ci-dessous plusieurs caractères dérivés qui semblent propres aux métazoaires.

Un premier trait commun à tous les métazoaires concerne la manière dont se déroule la méiose femelle. Ce type de méiose présente trois particularités. Premièrement, l'ovocyte interrompt la prophase de la première division méiotique par une pause plus ou moins longue, dont il profite pour s'accroître [16]. Deuxièmement, les chromosomes de l'ovocyte prennent un aspect particulier - dit en écouvillon - pendant la phase d'accroissement [17]. Troisièmement, les deux divisions méiotiques partagent de façon très inégale le cytoplasme de l'ovocyte. Celui-ci engendre une seule cellule de grande taille (l'ovule) et des cellules beaucoup plus petites, qui ne sont pas fonctionnelles (les globules polaires).

On trouve dans le répertoire des gènes d'autres caractères propres aux métazoaires. Par exemple, le proto-oncogène ets existe chez. les métazoaires, mais n'a pas été détecté chez d'autres organismes [18]. C'est également vrai pour le gène qui spécifie le collagène [19]. Ce gène serait apparu il y a 800 à 900 millions d'années, vers la même époque que les premiers métazoaires $[3,19]$. Il s'est ensuite dédoublé dans les diverses lignées animales, si bien que les chromosomes d'un métazoaire donné peuvent en contenir plusieurs copies [19]. On pense que le gène du collagène se constitua par duplications successives d'un module de 54 paires de bases. Chaque module spécifie six motifs de trois acides aminés, motifs répétés en multiples exemplaires dans la partie médiane de la molécule de collagène [20]. Un module correspond à un exon. Il y a de nombreux exons de 54 paires de bases dans les gènes qui gouvernent la synthèse d'une forme particulière de collagène chez une éponge et de nombreux vertébrés $[19,20]$. Il est probable que ce type d'organisation a été acquis par l'ancêtre commun de tous les métazoaires et conservé par certains d'entre eux [20].

\section{Caractères hérités par les métazoaires}

Peut-on préciser de quel type de cellules dérivent les métazoaires? Pour essayer de le savoir, il faut recenser les caractères partagés par l'ensem-

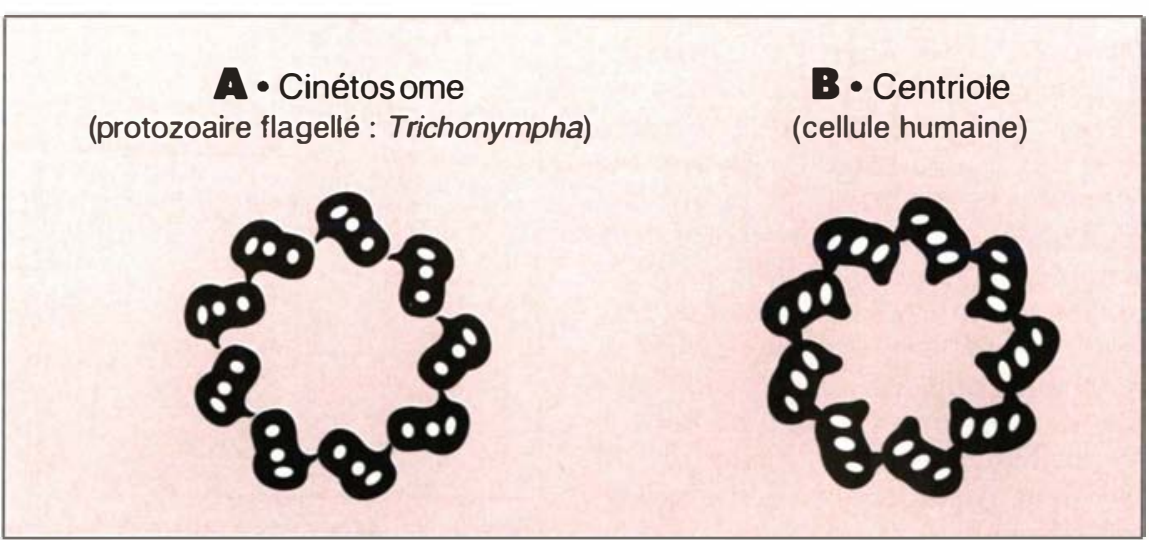

Figure 2. Similitude structurale entre un cinétosome et un centriole. La ressemblance se révèle quand on observe au microscope électronique des coupes transversales pratiquées dans le cinétosome situé à la base d'un flagelle (A) et dans l'un des deux centrioles qui constituent le centrosome (B). On voit une couronne de microtubules, éléments creux associés en triplets ou en doublets. Le cinétosome comporte 9 triplets qui se prolongent en autant de doublets à l'intérieur du flagelle. Le centriole comprend 9 triplets sur une partie de sa longueur et 9 doublets sur le reste. ( $D^{\prime}$ après des micrographies originales de G. Brugerolle (A) et Paintrand et al. [25] (B).) ble des métazoaires, et présents chez certains protistes. Ces caractères ancestraux devaient exister chez les précurseurs des métazoaires actuels.

Parmi les nombreux caractères communs aux protistes et aux métazoaires, trois nous paraissent importants. Ils concernent la reproduction sexuée et la locomotion des cellules. I.e premier trait est l'anisogamie, c'est-à-dire l'aptitude à produire des gamètes de taille inégale. La plupart des protistes sont anisogames, comme tous les métazoaires [21, 22]. l.e deuxième caractère commun est la prépondérance de la phase diploïde. Certains protistes et tous les métazoaires sont des diplontes: leur cycle vital comporte une phase diploïde prédominante; la phase haploïde se réduit à une courte période séparant la méiose de la fécondation [21, 22]. Le troisième point commun est la possession d'un flagelle. De nombreux protistes se déplacent grâce à un ou plusieurs flagelles qu'ils font onduler. La plupart des métazoaires produisent des spermatozoïdes qui se propulsent par le même moyen [11].

On ne peut pas certifier l'homologie des deux premiers caractères partagés par les protistes et les métazoaires, parce que les gènes qui les spécifient n'ont pas été clonés. Toutefois, le principe d'économie pousse à inclure ces traits communs parmi ceux que transmirent les protistes aux métazoaires. Quant aux flagelles, leur présence chez des organismes très différents doit être considérée comme un caractère homologue. Deux arguments appuient cette opinion. En premier lieu, l'axe du flagelle (l'axonème) est formé de deux protéines apparentées, dont la séquence varie peu suivant leur provenance : la tubuline $\alpha$ et la tubuline $\beta$ [23]. En second lieu, l'axonème possède une structure typique, observable aussi bien chez les protistes que chez certains végétaux et les métazoaires. Il comprend une couronne périphérique de neuf doublets de microtubules, disposés autour d'un doublet axial [24]. Dans le corps cellulaire, l'axonème se prolonge dans un élément figuré que l'on appelle ciné- 
tosome (figure 2). Un organite semblable se trouve à la base des cils $[24]^{*}$.

Les microtubules sont une innovation des protistes, dont les métazoaires ont hérité [15]. Ces éléments du cytosquelette jouent un rôle capital dans la vie des cellules. Leurs fonctions sont multiples [15]. Ils commandent notamment l'ondulation des flagelles ou des cils [24]. Ils guident aussi la migration des chromosomes. Entre ces deux types de mouvement, il y a donc une parenté évidente, que confirme l'intervention d'un élément commun dans la duplication des appareils moteurs : le centriole (figure 2). Tout cinétosome nouveau s'organise à partir d'un centriole qui s'est préalablement dédoublé. Chez certains protistes et les métazoaires, la séparation des chromosomes est précédée par la duplication des deux centrioles qui forment l'élément central du centrosome [25]. Chaque centrosome dédoublé sert de pôle de nucléation pour les microtubules qui constituent le fuseau mitotique [25].

Les cinétosomes des métazoaires sont incapables de se dupliquer quand ils occupent une position périphérique dans la cellule, à la base d'un cil ou d'un flagelle [15]. Une cellule ciliée ou flagellée ne peut donc pas se diviser [15, 26]. L'incompatibilité entre division et locomotion serait un caractère ancestral, acquis par les précurseurs directs des métazoaires [26]. Cette propriété persiste chez certains protistes actuels [15]. D'autres protistes (euglénidés, opalines, ciliés...) se divisent tout en conservant leurs appendices mobiles [15].

\section{Les précurseurs des métazoaires}

En fonction de ce qui précède, on peut esquisser un portrait-robot intégrant les principaux caractères que devaient réunir les ancêtres des

* Il n'y a pas de différence fondamentale entre un cil et un flagelle. Ces appendices sont quelquefois appelés "ondulipodes" [15, 26]. Entre un cil et un flagelle, la seule distinction que l'on peut faire concerne la longueur de l'onde qui les parcourt loms du battement. L'onde est plus courte que le flagelle, métazoaires actuels. Selon toute vraisemblance, il s'agissait de diplontes anisogames, mus par un ou plusieurs flagelles, mais incapables de se diviser tant que leur cytoplasme portait de tels prolongements.

Depuis longtemps, les choanoflagellés sont considérés comme les représentants actuels d'un groupe ancien qui donna naissance aux métazoaires [27]. Plusieurs observations appuient cette hypothèse. Selon une phylogénie moléculaire récemment publiée, les choanoflagellés seraient plus proches des métazoaires que ne le sont tous les autres protistes étudiés [9]. Par ailleurs, certains choanoflagellés forment des colonies de formes diverses, dont certaines ressemblent à des éponges [11, 26]. Enfin, les choanoflagellés ont un caractère commun avec les métazoaires les plus simples. Ils portent autour de leur unique flagelle une collerette qui sert à capturer la nourriture (figure 3). La collerette se compose d'une couronne de microvillosités, dont l'axe est formé par un faisceau d'actine [24]. On trouve chez les éponges des cellules à flagelle et collerette, que l'on appelle choanocytes. Ces cellules tapissent la paroi de l'endoderme. Elles ont une fonction nutritive [11, 12].

Il faut se garder de tirer des conclusions fermes concernant les affinités des choanoflagellés avec les métazoaires. Tout d'abord, il serait hasardeux de considérer comme un caractère homologue la possession d'une collerette par les choanoflagellés et les choanocytes. Ensuite, certains choanoflagellés peuvent se diviser sans rétracter leur flagelle [28]. Une autre remarque incite à la prudence : les choanoflagellés n'ont pas de sexualité connue [24]. Il est possible et même probable que les ancêtres lointains de ces organismes pratiquaient la reproduction sexuée. Toutefois, on ne peut pas affirmer qu'il s'agissait de diplontes anisogames, comme l'étaient probablement les précurseurs des métazoaires.

\section{La loi des récapitulations}

Comment se fit la transition initiale de l'état unicellulaire à l'état pluricellulaire? Un tel passage se renou- velle à chaque génération, lorsqu'un embryon se forme à partir d'un œuf. En quelque sorte, chaque animal refait en se développant ce que firent ses ancêtres en évoluant. Il y a donc une corrélation entre développement et évolution. Haeckel fit de cette correspondance un système, qu'il appela loi des récapitulations** ou loi biogénétique [1]. Formulée de façon simple, cette loi se réduit

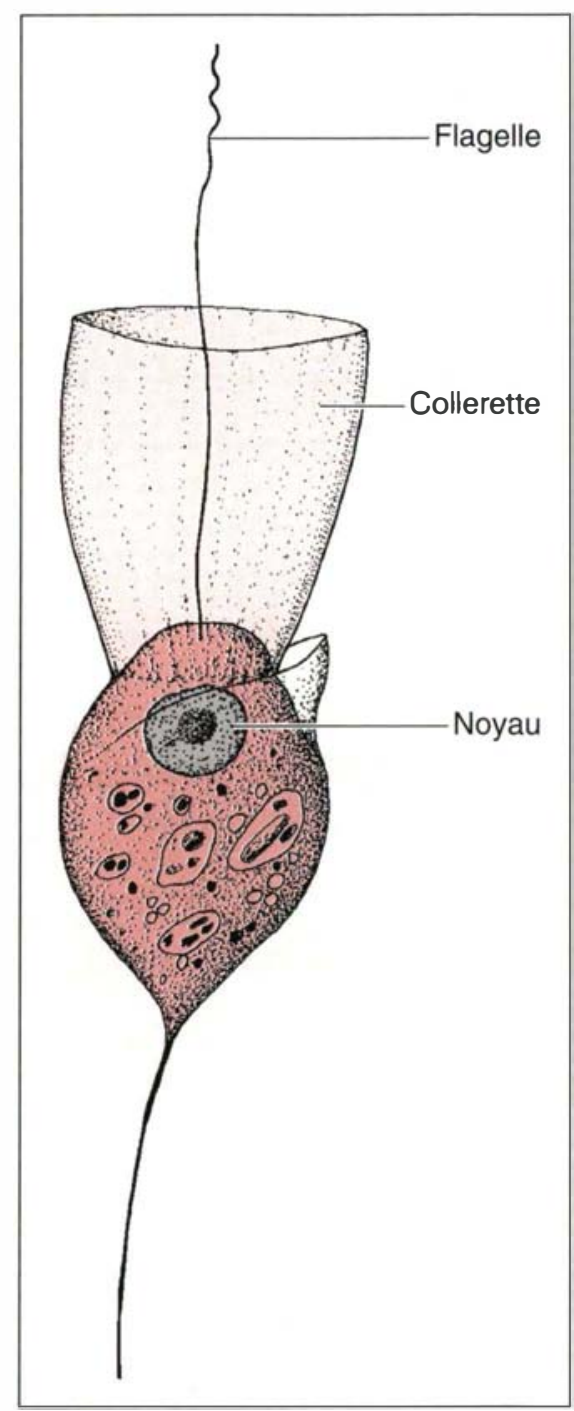

Figure 3. Structure d'un choanoflagellé typique. II s'agit d'une cellule de Sphaeroeca volvox, isolée à partir d'une colonie. La cellule est fixée par son pôle postérieur. A l'autre extrémité, elle porte un flagelle. Celui-ci est entouré d'une collerette formée de microvillosités unies par une gaine de nature glycoprotéique. 
à un slogan : l'ontogenèse récapitule la phylogenèse. En termes plus explicites, la théorie stipule qu'il existe une ressemblance entre les stades embryonnaires d'un animal donné et les formes adultes de ses ancêtres. En somme, l'embryon passe au cours de son développement par une série de stades qui miment les formes successives que revêtirent ses ancêtres au cours de l'évolution.

La théorie des récapitulations eut un énorme retentissement. Depuis sa publication, elle a constamment influencé la pensée des biologistes [29]. A son propros, les opinions se partagent entre adhésion nuan-

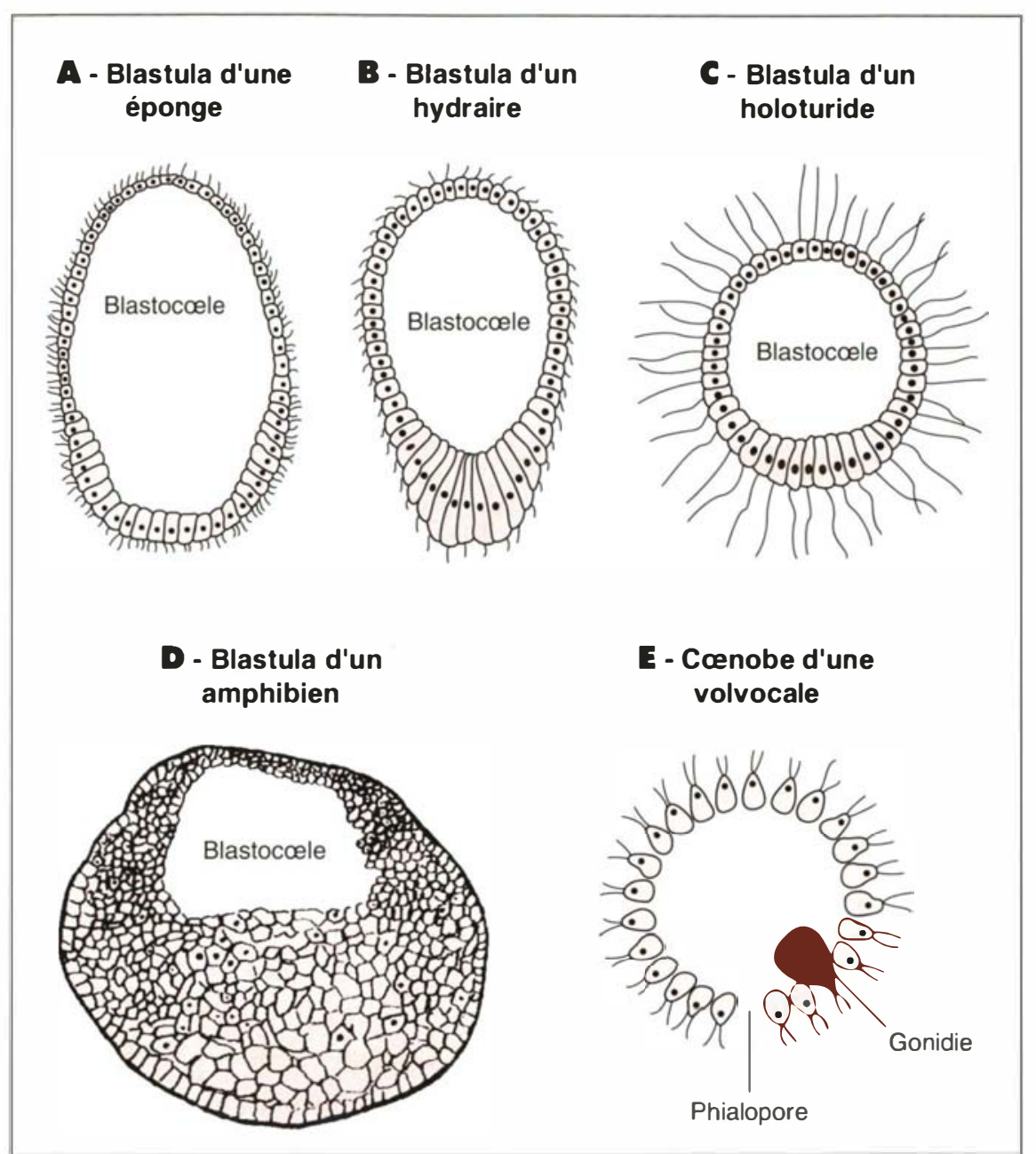

Figure 4. Comparaison entre blastulas et coenobe. Beaucoup d'animaux passent au début de leur développement par un stade similaire, que l'on appelle blastula. Après ce stade, l'embryogenèse suit des voies très divergentes. En effet, il n'y a aucune ressemblance entre les formes adultes d'une éponge (A), d'un cnidaire (B), d'un échinoderme (C) et d'un vertébré (D). Une blastula typique $(A-D)$ a la forme $d^{\prime}$ une sphère ou $d^{\prime}$ un sphéroïde. Elle comporte une ou plusieurs strates de cellules délimitant une cavité interne, nommée blastocoele. Sur leur face exposée au milieu extérieur, les cellules portent un ou plusieurs cils. La blastula des animaux ressemble à un coenobe de Volvox (E), qui est une colonie de cellules flagellées. Contrairement à la blastula, le coenobe possède un orifice (le phialopore) et comprend deux catégories de cellules, dont les plus grandes (les gonidies) donneront nais sance aux gamètes. Les schémas ne sont pas à la même échelle. Le grossissement utilisé rend invisible la ciliature qui recouvre la surface de la blastula d'amphibien. (Schéma A : d'après Grassé et al. [12]. Schéma B : d'après Buss [26]. Schéma C : d'après Hyman [11]. Schéma D: d'après Grassé et Devillers [32]. Schéma E : d'après Mignot [33].) cée $[6,11,26]$ et franche opposition $[13,30,31]$. Un siècle de débats a sérieusement entamé la crédibilité de la loi biogénétique. L'ontogenèse ne répète pas exactement les événements évolutifs. Il y a des étapes évolutives que le développement semble allonger, raccourcir et même supprimer [26, 29, 30]. Certains stades embryonnaires n'ont apparemment aucun équivalent phylogénétique [26, 30]. Selon toute vraisemblance, les embryons des organismes actuels ressemblent plus aux formes embryonnaires qu'aux formes adultes de leurs ancêtres présumés [29].

Mais un aspect de la théorie résiste mieux à un examen critique : l'embryogenèse respecte la succession des événements évolutifs. Cette constatation ne fait que trahir une évidence : la complexité des organismes augmente à mesure qu'ils se développent, comme elle augmenta à mesure qu'ils évoluèrent. Si le développement conserve souvent l'ordre supposé des événements évolutifs, c'est que beaucoup de processus embryonnaires sont liés par une relation de cause à effet, qui leur impose de se dérouler suivant une séquence déterminée. Toutefois, certaines lignées animales ont apparemment modifié la succession ancestrale des événements embryonnaires. De tels changements sont appelés hétérochronies [26, 29, 30]. Par exemple, certains animaux retardent le développement de leurs différents organes par rapport à celui des gonades: ils deviennent néoténiques [29].

Appliquant la loi biogénétique, Haeckel fit une supposition simple concernant la structure des premiers métazoaires. Il remarqua que l'œuf de nombreux animaux se divise en cellules de plus en plus petites, qui se disposent en une sphère ou un sphéroïde creux (figure 4). Ce stade

** Ce n'est pas Haeckel qui a inventé le concept de récapitulation [29]. Suivant certains biologistes influents du débnut du XIX'siècle (Oken, Meckel), les animaux reproduisent en se développant les formes adultes d'animaux situés à un stade inférieur dans l'échelle d'organisation. Haeckel maria cette idée à celle d'évolution. I'après lui, les onganismes áoluent en acquérant des carcuctères nouveaux. Ceux-ci s'intègrent au développement par un processus d'addition teminale. Il y a un lien mécanique entre phylogenèse et ontogenèse [1]. 
embryonnaire est appelé blastula. Une garniture de cils permet à l'embryon de se déplacer. Selon Haeckel, la blastula représente l'équivalent embryonnaire d'un animal primitif, la blastaea [2]. Tous les métazoaires dériveraient donc d'un ancêtre de ce type. Cette proposition nous paraît raisonnable.

\section{Les protistes coloniaux}

Pendant la phase végétative de leur cycle vital, certains protistes élaborent des colonies qui ressemblent à des blastulas. Cela incite à penser que des protistes coloniaux furent à l'origine des métazoaires. Ces derniers seraient l'aboutissement le plus achevé d'une tendance évolutive répandue parmi les protistes: la propension à vivre en société. Chez les seuls flagellés, au moins trois groupes majeurs comportent des espèces coloniales [21, 24]. I.es colonies les plus complexes sont les coenobes que construisent les volvocales (figure 4).

I es coenobes ont avec les métazoaires plusieurs traits communs [11, 24, 33]. Parmi ceux-ci, trois nous paraissent essentiels : l'adhérence, la communication et la spécialisation cellulaires [34]. Quelles formes revêtent dans les coenobes ces divers phénomènes? La cohésion est assurée par une sorte de gangue de nature glycoprotéique, englobant les cellules. Celles-ci n'entrent pas en contact par leur membrane plasmique [33]. En cela, les coenobes se distinguent nettement des métazoaires, dont les cellules forment de vastes ensembles (les tissus et les organes), où elles sont généralement accolées les unes aux autres. Dans les coenobes, la communication et la spécialisation sont réduites à leur plus simple expression. Les cellules communiquent principalement par des ponts cytoplasmiques [33]. Elles se différencient en deux catégories, somatique et germinale. Les unes remplissent les fonctions de ménage, comme la locomotion et la phototaxie. Les autres, moins nombreuses, se chargent de la reproduction [33]. Malgré certaines ressemblances avec les métazoaires, on ne saurait rapprocher les volvocales des ancêtres res, et ce pour plusieurs raisons. En premier lieu, les volvocales sont des haplontes [33], tandis que les métazoaires sont des diplontes [22]. En deuxième lieu, il s'agit d'organismes végétaux, dont les cellules renferment un chloroplaste [21]. Enfin, les phylogénies moléculaires tendent à rattacher les phytoflagellés - dont font partie les volvocales - aux plantes vertes plutôt qu'aux métazoaires [9, 35].

\section{L'adhérence cellulaire}

Chez les métazoaires, l'adhérence entre cellules fait intervenir de nombreuses molécules. Cette complexité s'explique par le fait que les membranes plasmiques n'ont aucune adhérence intrinsèque. Ia cohésion des tissus et des organes dépend de deux types d'interactions: cellulescellules et cellules-matrice extracellulaire. Ces interactions sont assurées par des protéines transmembranaires. Les cellules adhèrent les unes aux autres grâce à des protéines qui se lient par leur domaine externe: cadhérines, intégrines, CAM (cell adhesion molecules), etc. [36]. Les intégrines attachent également les cellules à la matrice extracellulaire, réseau de fibrilles composé de glycosaminoglycanes, de protéoglycanes et de protéines (collagène, fibronectine, laminine, tenascine...) [36]. A l'intérieur des cellules, les intégrines sont fixées au cytosquelette, et notamment aux microfilaments d'actine [37].

Certaines molécules adhésives ne se contentent pas d'assurer la cohésion de l'organisme. Elles peuvent aussi influencer la multiplication et la différenciation des cellules quand celles-ci adhèrent par leur entremise à d'autres cellules ou à la matrice extracellulaire [38, 39]. I es intégrines participent à cette forme de signalisation [39].

Pour assurer la cohésion entre leurs cellules, les métazoaires durent innover, donc acquérir de nouveaux gènes. Il n'est pas raisonnable de penser que ces gènes ont été forgés de toutes pièces. Nous supposons qu'ils ont été créés par duplication de gènes préexistants, qui avaient en charge des fonctions différentes chez les protozoaires. Mais, à notre connaissance, on n'a découvert chez les protistes aucun gène qui présentât une parenté incontestable, permettant de conclure à une homologie, avec les gènes qui spécifient les diverses protéines adhésives des métazoaires [34].

Une telle homologie est peut-être à rechercher dans la famille des intégrines. Ces protéines pourraient intervenir dans un processus très ancien, bien antérieur à l'apparition des métazoaires: la fusion des gamètes (syngamie). On pense qu'une intégrine permet à l'ovule des mammiferes de fusionner avec le spermatozoïde en se liant à une molécule superficielle de celui-ci, appelée $\mathrm{PH}-30$ [40]. Il est possible que les métazoaires aient réemployé, pour faire adhérer leurs cellules somatiques, des protéines déjà utilisées par les protistes pour faire adhérer, puis fusionner leurs cellules sexuelles.

\section{La communication cellulaire}

Les membranes plasmiques sont des isolants efficaces. Les cellules des métazoaires communiquent avec leur environnement de deux façons: par contact ou par messages. La communication par contact s'établit grâce à des protéines membranaires qui se lient aux cellules voisines ou à la matrice extracellulaire [39, 41$]$. La communication par messages fait intervenir des signaux variés: facteurs de croissance, inducteurs, attractants, hormones, neurotransmetteurs. Ces signaux agissent respectivement sur la multiplication, la différenciation, les déplacements, le métabolisme et le contenu en ions des cellules. La communication se fait entre cellules émettrices et cellules réceptrices. L'émission du signal consiste en une sécrétion. Sa réception a lieu à l'intérieur ou à la surface des cellules cibles. Il y a donc deux grandes classes de récepteurs: les récepteurs intracellulaires, qui perçoivent les signaux capables de pénétrer dans les cellules, et les récepteurs membranaires, qui perçoivent les signaux incapables de traverser les membranes [42].

Les récepteurs intracellulaires sont localisés dans le noyau. lls sont donc appelés nucléaires. Ces récepteurs 


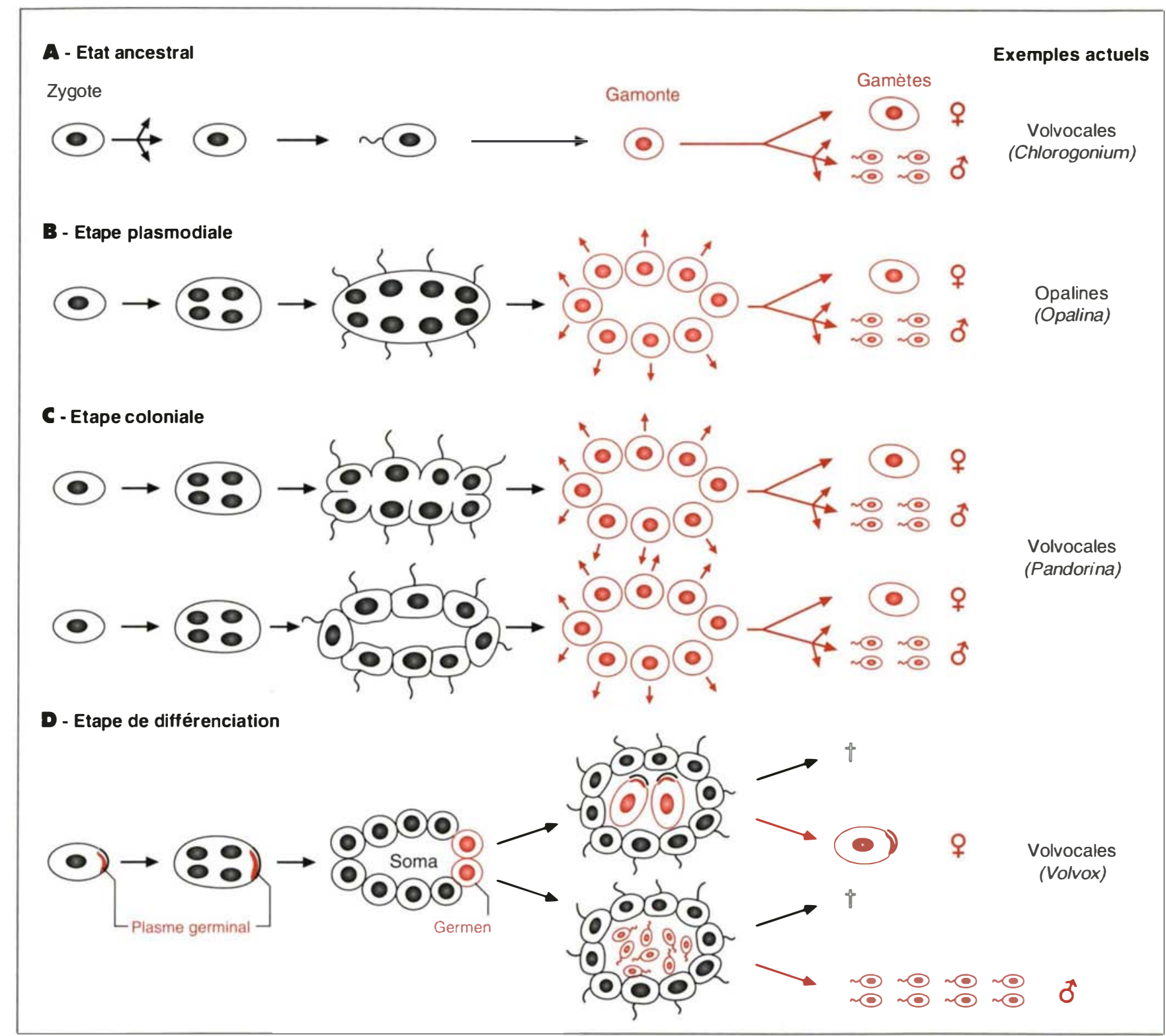

Figure 5. Évolution des protozoaires en métazoaires. Nous postulons plusieurs étapes dans l'évolution des protozoaires flagellés (A) en métazoaires primitifs (D). Chez les protozoaires (A), le cycle vital fait alterner une phase végétative (figurée en noir) avec une phase sexuée (figurée en rouge). Au cours de la phase végétative, les cellules se multiplient par mitose et se différencient. Elles acquièrent notamment un flagelle. Pendant la phase sexuée, les cellules se convertissent en gamontes, qui se transforment en gamètes femelles (immobiles) et en gamètes mâles (mobiles). L'étape plasmodiale (B) constitue un premier pas dans l'évolution vers l'état pluricellulaire. Le noyau du zygote se divise, mais la partition du cytoplasme est retardée jusqu'au début de la phase sexuée. A ce stade, la cellule plurinucléée (plasmode) se fragmente en gamontes. L'étape coloniale (C) commence quand la cellularisation du plasmode devient plus précoce et survient pendant la phase végétative. Si le changement est progressif, on obtient d'abord un groupe de cellules communiquant par leur cytoplasme, puis une colonie de cellules identiques. L'étape de différenciation (D) marque l'avènement d'une embryogenèse. Le cytoplasme du zygote se régionalise en acquérant un plasme germinal, apporté par le gamète femelle. Le plasme germinal est transmis à certaines cellules issues du zygote. Ces cellules forment le germen et donneront naissance aux gamètes. Toutes les autres cellules édifient le soma, qui devient mortel. 
sont tous organisés de la même façon [43]. Ils comportent notamment un site apte à fixer le messager chimique (stéroïde, dérivé de la vitamine $\mathrm{A}$, ou autre ligand hydrophobe) et un domaine activateur. Celui-ci stimule la transcription de certains gènes lorsqu'un ligand approprié occupe le site de liaison [43].

Il existe au moins deux types de récepteurs membranaires. Tous deux comportent un domaine extracellulaire, qui perçoit le signal, un domaine membranaire et un domaine intracellulaire, qui traduit le message en termes intelligibles pour la cellule [42]. A la première catégorie se rattachent les récepteurs de l'insuline et de plusieurs facteurs de croissance. Les récepteurs de ce type transmettent directement les signaux extracellulaires [42]. Leur domaine interne a une activité enzymatique de type tyrosine kinase. Celle-ci est stimulée lorsqu'un ligand adéquat occupe le récepteur. I.a kinase active à son tour un réseau très complexe d'enzymes qui modifient la phosphorylation, donc l'activité, de nombreuses protéines intervenant notamment dans la transcription et la traduction [44].

Les récepteurs de la seconde catégorie perçoivent des messages de nature très variée: photons, odeurs, hormones, neurotransmetteurs [34]. Ils ont ceci de remarquable que la protéine qui les forme possède sept segments transmembranaires. Ces récepteurs transmettent les signaux extracellulaires grâce à un système de transduction, qui consiste en une protéine liée à la face interne de la membrane plasmique. Cette protéine, dite $G$, comporte trois sousunités, dont l'une s'associe au GTP ou au GDP [42]. On connaît plusieurs espèces de protéines $\mathrm{G}$. Leur action s'exerce sur de nombreux processus cellulaires. Elles peuvent, soit activer le réseau de kinases évoqué plus haut [44], soit commander l'ouverture de canaux ioniques, soit modifier la concentration de divers messagers intracellulaires: AMPc, GMPc, inositol triphosphate, diacylglycérol, ions $\mathrm{Ca}^{2+}[42]$.

Dans le domaine de la communication, les métazoaires n'ont pas tout inventé. Certains mécanismes préexistaient chez les organismes unicellulaires. En effet, on a mis en évidence chez la levure un système de communication complet, homologue de celui des animaux, ainsi que des éléments de deux autres systèmes: celui qui recourt à des récepteurs de type tyrosine kinase, et celui qui recourt à des récepteurs nucléaires. Le premier système fait intervenir des récepteurs liés à des protéines G. Grâce à lui, les cellules de levure échangent des signaux de nature peptidique (les phéromones), qui les préparent à la syngamie [45]. La levure possède aussi une cascade d'enzymes qui, chez les métazoaires, traduit les messages perçus par les récepteurs de type tyrosine kinase [44]. Enfin, la levure peut intégrer des récepteurs nucléaires d'origine animale. Elle devient alors capable de répondre à l'administration de ligands appropriés, tels que les stéroïdes, en faisant varier l'activité de certains gènes [46]. C'est donc que les récepteurs utilisés par les métazoaires peuvent reconnaître des gènes de levure et s'intégrer à leur appareil de transcription. Il est probable que les récepteurs nucléaires sont des éléments modifiés d'un système de transcription très ancien.

\section{L'étape plasmodiale}

Chez les protistes solitaires se reproduisant de façon sexuée, deux programmes génétiques sont exprimés en alternance (figure 5). L'un gouverne la vie végétative, pendant laquelle les cellules se multiplient par mitose. L'autre gouverne la phase sexuée du cycle vital, qui convertit les cellules végétatives en gamètes. Pour construire un organisme multicellulaire, il est nécessaire de modifier ces deux programmes. Le programme végétatif doit être transformé de manière à réunir et à faire vivre ensemble un groupe de cellules. Le programme sexué doit aussi être modifié. Une exigence subsiste, qui est de libérer les gamètes au moment de la reproduction.

De nombreux gènes contrôlent chaque phase du cycle vital. Beaucoup durent être dédoublés et modifiés lors de l'acquisition des fonctions propres aux métazoaires, comme l'adhérence et la communication entre cellules. Il nous paraît donc difficile d'imaginer un passage direct du stade unicellulaire au stade colonial, créant des communautés mobiles de cellules accolées par leur membrane plasmique. Nous proposons une étape intermédiaire, qui ferait intervenir des cellules plurinucléées, que l'on appelle plasmodes. Cette idée n'est pas neuve. Elle fut proposée à plusieurs années d'intervalle par différents auteurs [47, 48]. Plusieurs arguments nous paraissent justifier l'existence d'une étape transitoire de type plasmodial (figure 5). Un plasmode a un avantage sélectif évident: il peut devenir beaucoup plus grand qu'une cellule uninucléée, tout en restant apte à se mouvoir. Le maintien de la motilité nous semble crucial. Beaucoup de protistes sont apparemment passés de l'état unicellulaire à l'état colonial en façonnant une sorte de colle qui empêche leurs cellules de se disperser. Mais cette gangue isole les cellules et les immobilise. De fait, de nombreux protistes perdent leur motilité quand ils deviennent coloniaux $[21,24]$. Cils ou flagelles servent alors à la nutrition.

Une autre raison milite en faveur du passage par un intermédiaire plasmodial lors de la formation des colonies mobiles. La double transformation (cellule solitaire $\rightarrow$ plasmode $\rightarrow$ colonie) peut être entraînée par un simple changement de chronologie, cause présumée de nombreuses innovations évolutives [29, 30]. Ce changement affecterait le déroulement de la mitose, en découplant la division du cytoplasme (cytocinèse) de celle du noyau (caryocinèse). Dans un premier temps, la cytocinèse serait retardée, si bien que le zygote engendre un plasmode (figure 5). Au début de la phase sexuée, le plasmode se fragmente en gamontes, qui se différencient en macrogamètes et en microgamètes.

Il existe des formes plurinucléées dans plusieurs groupes de protistes: amibes, héliozoaires, foraminifères, opalines, ciliés [11, 21, 24]. Par exemple, les opalines sont plurinucléées pendant la phase végétative 
de leur cycle vital, mais elles produisent des gamètes uninucléés [21, 24]. Chez certains métazoaires, le noyau de l'œuf se divise, sans que le cytoplasme se scinde. On obtient ainsi un embryon plurinucléé, qui est un syncytium. C'est ce qui se produit au cours du développement des insectes [12]. Dans plusieurs groupes zoologiques (éponges hexactinellides, gastérotriches, rotifères, nématodes), l'adulte possède une organisation syncytiale qui concerne, soit la totalité de l'organisme, soit une partie de ses tissus [12, 49].

\section{L'étape coloniale}

Il paraît facile de convertir un plasmode en colonie. Il suffit qu'un nouveau changement de chronologie fasse apparaître des membranes autour des noyaux végétatifs (figure 5). Nous supposons que la cellularisation s'est faite de façon progressive: des cloisons cellulaires se forment, mais ne se ferment pas tout à fait. Cela maintient, comme dans les colonies de volvocales, une continuité cytoplasmique, qui aide à la cohésion de l'ensemble et coordonne ses diverses fonctions.
Pour se transformer en colonies, les plasmodes durent acquérir des molécules adhésives, qui empêchent les cellules de se disperser. On peut penser que ces molécules apparurent avant même que les cloisons cellulaires ne se ferment complètement. Cette idée implique que dans une étape de transition entre plasmodes et colonies, la cohésion des organismes était assurée à la fois par des ponts cytoplasmiques et par des molécules adhésives. Une fois les ponts obturés, ces dernières suffisaient à maintenir l'unité de la colonie.

La structure de la colonie dépend de la force des interactions cellulaires. Nous pensons que che\% les ancêtres coloniaux des métazoaires les cellules ne portaient à leur surface qu'un nombre restreint de molécules adhésives. Une double conséquence en découle. Les molécules adhésives se regroupent aux interfaces entre cellules, si bien que celles-ci forment un épithélium, au lieu de s'agréger en une masse compacte (figure 6). Ce facteur est l'un de ceux qui contribuent à créer une colonie creuse, semblable à la blastaea de Haeckel [50]. Une telle

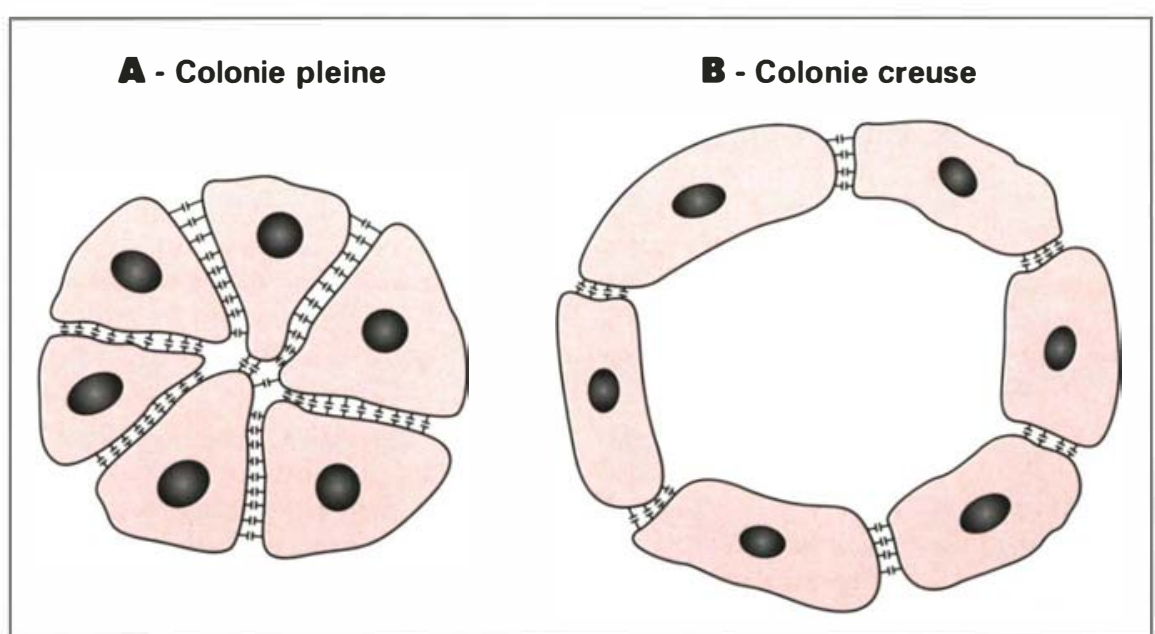

Figure 6. Relations entre les cellules d'une colonie. On peut supposer que chez les ancêtres coloniaux des métazoaires actuels, la cohésion entre les cellules était assurée par des molécules adhésives. Selon toute probabilité, la forme de la colonie dépendait du nombre de molécules adhésives que portait à sa surface chaque cellule de la colonie. Si ces molécules sont nombreuses, les cellules ont une forte affinité les unes pour les autres. Elles forment un amas compact, dépourvu de cavité (A). Si les molécules adhésives sont dispersées, elles se concentrent à l'interface entre les cellules voisines. La colonie se distend et prend la forme d'une sphère ou d'un sphéroïde creux (B).

colonie a un avantage sélectif par rapport à un plasmode: elle dispose d'ine plus grande surface de contact avec le milieu extérieur, ce qui facilite la respiration et l'évacuation des déchets métaboliques. Nous considérons la cavitation comme une étape évolutive importante. Elle se répète pendant l'embryogenèse de nombreux animaux (figure 4).

En matière de communication, tout progrès semble à première vue bloqué au stade plasmodial. Tant qu'elles restent unies par des ponts cytoplasmiques, les cellules n'ont apparemment aucun besoin de développer un système de communication transmembranaire. Toutefois, rien n'exclut qu'à l'intérieur d'un plasmode les zones éloignées puissent communiquer par messages et récepteurs intracellulaires. Ce système ne devrait pas être affecté par l'occlusion des parois cellulaires car il utilise des signaux hydrophobes, capables de traverser les membranes. Ainsi, les cellules peuvent continuer à dialoguer après qu'elles se sont isolées complètement. Toutes se comportent à la fois comme émettrices et comme réceptrices des signaux coordinateurs. Ce type de communication ne réclame aucune différenciation cellulaire.

Les plasmodes et les colonies conservèrent l'usage du système de communication par récepteurs liés aux protéines G. Ce système est d'origine très ancienne. Il devait être utilisé pendant leur phase sexuée par les précurseurs successifs des métazoaires (figure 5). Cet emploi subsiste chez des organismes aussi différents que la levure [45] et les échinodermes [31]. Chez ces derniers, la fécondation active apparemment une protéine $G$, ce qui entraîne de nombreuses modifications dans le cytoplasme de l'œuf [31].

\section{La différenciation des colonies}

Un plasmode cellularisé est une colonie. Sous sa forme la plus simple, une colonie est formée de cellules identiques et son cycle vital ressemble à celui d'un protiste (figure 5) [21, 24]. Pendant la phase sexuée, tous les éléments de la communauté se transforment en gamon- 
tes. C'est ce qui se produit notamment chez Pandorina [33]. Pour convertir un organisme colonial en métazoaire, il faut faire apparaître une différenciation parmi les cellules issues du zygote. En d'autres termes, il faut inventer une embryogenèse.

Quelle différenciation apparut en premier lieu? Nous postulons une diversification initiale des cellules en deux catégories, somatique et germinale. Deux considérations soutiennent cette proposition. Premièrement, la dichotomie soma-germen s'instaure très précocement pendant l'embryogenèse de nombreux animaux $[26,51]$. En vertu de la loi biogénétique, cela pourrait refléter un événement évolutif très ancien. Le second argument se réfère au cycle vital des protistes solitaires et coloniaux. Chez ces organismes, des cellules de même lignée mettent successivement en ouvre deux programmes génétiques distincts (figure 5). $\mathrm{Si}$, dans une colonie, des cellules différentes exécutent ces mêmes programmes, on aura un début de spécialisation cellulaire. Un soma et un germen apparaissent. C'est ce qui se produit chez certaines volvocales comme Pleodorina et Volvox [33].

Les cellules du germen conservent plusieurs propriétés ancestrales, que possédaient les protozoaires et les gamontes engendrés par les protistes coloniaux. En premier lieu, ces cellules n'ont aucune affinité les unes pour les autres, ni pour les cellules somatiques. Elles ont donc tendance à pénétrer dans la cavité que forme le soma (figure 5). En deuxième lieu, les cellules germinales sont aptes à se convertir en gamètes, donc à subir la méiose et à garnir leur surface de molécules qui leur permettront de se reconnaître, puis de fusionner [22]. Enfin, les cellules germinales sont potentiellement immortelles: elles peuvent proliférer à l'infini, à condition de rencontrer un partenaire de type sexuel opposé [51].

Quel avantage y avait-il pour les métazoaires archaïques à confier la fonction reproductrice à un lot restreint de cellules? Le bénéfice devait être immédiat, et l'emporter était de réduire le nombre de cellules capables de subir la gamétogenèse. L'avantage consistait peut-être à fournir une protection aux cellules reproductrices. Quand une colonie entre en phase sexuée, toutes ses cellules se transforment en gamontes. Elles doivent donc se dédifférencier en perdant leur flagelle et leurs molécules adhésives, puis se convertir en ovules ou en spermatozoïdes (figure 5). Pendant qu'elles se modifient, les cellules sont particulièrement exposées à l'attaque des prédateurs. S'il existe un germen, la gamétogenèse se déroule à l'intérieur du soma. Ce dernier protège les cellules germinales en cours de maturation, tout en continuant à accomplir les fonctions végétatives (locomotion, nutrition...). A plus long terme, un autre avantage apparait. Les cellules germinales femelles (ovocytes) peuvent s'accroitre en absorbant des substances nutritives fournies par le soma [16]. Cela augmente la taille et par conséquent les chances de survie du zygote. Dans une colonie, pareil accroissement est impossible puisque la taille du zygote est limitée par celle du gamonte qui l'engendre, donc par celle de chaque cellule formant la communauté (figure 5).

\section{Le plasme germinal}

Pour créer un soma et un germen, il faut modifier le cytoplasme du zygote. Celui-ci doit concentrer en un lieu particulier des facteurs aptes à faire exécuter le programme sexué par certaines cellules de l'embryon. Ces facteurs constituent le plasme germinal [51]. Ils sont élaborés par l'ovocyte et restent en place dans l'ouf. Nous pensons que chez les métazoaires archaïques, le plasme germinal était localisé en périphérie du zygote, et associé au cortex ovulaire. On retrouve une telle association dans les groupes zoologiques où un plasme germinal a pu être mis en évidence [16]. Ce sont donc des cellules situées en périphérie de l'embryon qui acquièrent le plasme germinal et deviennent gonocytes [16].

A nos yeux, c'est l'acquisition d'un plasme germinal qui distingue les métazoaires de leurs ancêtres coloniaux. Bien que le plasme germinal soit apparemment d'origine très ancienne, il ne peut être détecté que dans quelques groupes zoologiques $[26,51]$. Par ailleurs, la lignée germinale est difficile à déceler chez certains animaux tels que les cnidaires. Toutefois, les cellules sexuelles y dérivent non pas de cellules différenciées, mais de cellules souches qui restent totipotentes $[52,53]$. En somme, ces cellules retardent leur engagement dans la voie germinale ou somatique. C'est un exemple d'hétérochronie.

En faisant apparaître un plasme germinal dans leurs gamètes femelles, les métazoaires archaïques ne firent qu'utiliser une propriété déjà acquise par les protozoaires. Chez ces animaux, le cytoplasme est visiblement polarisé. C'est manifeste pour ce qui concerne les flagellés. Les ciliés possèdent même des facteurs cytoplasmiques, dont le rôle évoque celui d'un plasme germinal. Chez Paramecium tetraurelia, la méiose produit huit noyaux, dont sept dégénèrent. Une zone proche de la bouche (le cône adoral) protège contre la destruction le noyau haploïde qui s'y trouve [21]. Après la conjugaison, le noyau zygotique se divise deux fois. Sur les quatre noyaux fils, deux deviennent micronuclei et deux macronuclei [21]. Ceux-ci ne se forment que dans la partie postérieure du zygote [54]. Il y a donc en périphérie de la cellule deux régions douées de propriétés particulières, qui orientent l'évolution des noyaux qu'elles contiennent. On ignore la nature des facteurs qui matérialisent cette influence. Ces facteurs sont probablement liés au cytosquelette.

Ce qui se produit chez la paramécie a très bien pu se passer chez les précurseurs des métazoaires. Mais cela ne donne aucune indication sur la nature des déterminants germinaux. Chez l'animal le mieux étudié à cet égard (la drosophile), on ne connaît pas encore bien le mode d'action de ces déterminants [55]. En tout cas, de nombreux gènes gouvernent leur synthèse puis leur localisation au pôle postérieur de l'ovocyte [55]. Plusieurs ARN messagers sont localisés dans cette région 
de la cellule. L'un d'eux (le produit de nanos) a un intérêt particulier, bien que le gène nanos ne soit apparemment pas requis pour la formation du plasme germinal [55]. Il existe un homologue présumé de nanos chez les amphibiens anoures [56]. L'ARN correspondant est concentré dans une zone de l'ovocyte (le cortex végétatif) [56], où résident les déterminants germinaux [51]. Des mécanismes semblables pourraient donc présider à la régionalisation de l'ovocyte dans des groupes aussi différents que les arthropodes et les vertébrés.

\section{Propriétés du soma}

Les cellules d'un organisme colonial sont soumises à de multiples contraintes: se multiplier et se différencier pendant la phase végétative du cycle vital; se dédifférencier et se convertir en gamètes durant la phase sexuée; proliférer de nouveau après la fécondation (figure 5). Après l'acquisition d'un germen, les choses changent. N'ayant plus à se transformer en gamètes, les cellules somatiques peuvent se différencier sans conserver ou recouvrer l'aptitude à proliférer. De fait, prolifération et différenciation sont en partie disjointes durant l'embryogenèse des animaux actuels. Les cellules commencent par se multiplier. Elles subissent ensuite diverses modifications morphologiques (comme l'acquisition d'une garniture ciliaire), qui sont souvent incompatibles avec la poursuite des mitoses. Incapables de se diviser, les cellules différenciées sont condamnées à périr.

La plupart des métazoaires ont un soma mortel. C'est vrai même pour ceux qui se reproduisent de façon asexuée, par bourgeonnement ou par scissiparité $[57,58]$. Puisqu'elle est très répandue, la mortalité est probablement un caractère ancestral, acquis par les métazoaires archaïques et conservé par leurs descendants. Mais cela ne nous dit pas quel était pour les métazoaires primitifs l'avantage immédiat d'une telle innovation. On ne peut avancer que des hypothèses. C'était peutêtre un procédé simple et efficace pour limiter la taille du soma. De la sorte, il devenait impossible de construire des organismes trop grands, donc non viables parce qu'incapables de coordonner l'activité de leurs cellules [51].

Si la mortalité a une origine très ancienne, elle pourrait avoir une cause unique chez tous les métazoaires. Est-ce réellement le cas? Il est difficile de répondre à cette question. Tout d'abord, les études de mortalité se sont concentrées sur quelques groupes zoologiques. Ensuite, la mortalité est régie par des mécanismes complexes, agissant sur la cellule et sur l'organisme tout entier. Chez les vertébrés, les cellules somatiques ont une mortalité intrinsèque. Leur capacité de prolifération est réduite : après un nombre déterminé de mitoses, elles deviennent sénescentes. Quant au soma en tant que tel, sa longévité dépendrait de nombreux gènes dont l'action conjuguée optimise la durée de la vie $[59,60]$. C'est apparemment le cas pour les vertébrés: aucun gène ne semble à lui seul capable de moduler la longévité du soma. Certes, on connaît quelques gênes - comme celui qui est responsable du syndrome humain de Werner - dont l'activation suffit pour entraîner un vieillissement précoce de l'organisme entier [61]. Mais quand il est muté, ce gène paraît agir en accélérant la sénescence individuelle des cellules [62]. Une théorie en vogue stipule que les gènes dont dépend la longévité du soma auraient une influence bénéfique sur la survie de l'organisme quand il est jeune, mais néfaste lorsqu'il vieillit $[59,60]$. De tels gènes pourraient exister parce que la sélection naturelle n'élimine pas les mutations délétères affectant le soma après la période de reproduction. En somme, stérilité et grand âge équivalent, du point de vue sélectif, à la mort [59].

\section{Conclusion}

Il est probable que la dichotomie germen-soma s'instaura chez les métazoaires les plus primitifs. Ce fut une manière simple d'adapter la reproduction sexuée à l'état pluricellulaire. En acquérant un germen, les premiers métazoaires firent un choix décisif : interdire à certaines de leurs cellules l'accès direct à la fonction reproductrice. A posteriori, ce choix se révéla fructueux car il ouvrait la voie à une différenciation de plus en plus poussée du soma. Mais la différenciation impose des limites à la prolifération des cellules somatiques, donc à la régénération et à la reproduction asexuée. Celle-ci n'est possible que si l'organisme adulte conserve certaines de ses cellules dans un état indifférencié, semblable à celui des premiers blastomères. C'est ce qui se produit dans quelques groupes zoologiques, où coexistent les deux modes de reproduction, sexué et asexué. Ces groupes estompent la distinction ancestrale entre germen et soma

\section{RÉFÉRENCES}

1. Haeckel E. (jenerelle Morphologie der Orgat nismen. Berlin : Reimer, 1866.

2. Haeckel E. Die Gastraea-Theorie, die phylogenetische Klassifikation des Tierreiches und Homologie der Keimblätter. Jenaische Z Nalurwiss 1874; 8: 1-55.

3. Conway Morris S. The fossil record and the early evolution of the Metazoa. Nalure $1993 ; 361: 219-25$.

4. Cloud P, Glaessner MF. The Ediacaran period and system: Metazoa inherit the earth. Science 1982; 217: 783-92

5. Hennig $\mathrm{W}$. Cimundzüge einer Theorie der phylogenetischen Systematik. Berlin : Deutscher Zentralverlag, 1950.

6. Hanson EI). The origin and early evolution of animals. Middleton: W'esleyan University Press, 1977.

7. Field K(i, Olsen (j], Lane I)J, (Giovannoni SJ, Ghiselin MT, Raff EC, Pace NR, Raff RA. Molecular phylogeny of the animal kingdom. Science 1988; 239: 748-53. 8. Lake JA. Origin of the Metazoa. Proc Nall Acad Sci USA 1990; 87: 763-6.

9. Wainright PO, Hinkle (;, Sogin ML, Stickel SK. Monophyletic origins of the Metazoa: An evolutionary link with fungi. Science 1993 ; 260 : 340-2.

10. Adoutte A, Philippe $H$. The major lines of metazoan evolution: summary of traditional evidence and lessons from ribosomal RNA sequence analysis. In : Pichon Y, ed. Comparative molecular neurobiology. Bäle: Birkhäuser, 1993: 1-30.

11. Hyman LH. The Invertebrales: Protozoa through Ctenophorc. New York: McCiraw-Hill, 1940 .

12. Grassé PP, Poisson RA, Tuzet (). Zoologie. Tome 1. Inverlébrés. Paris: Masson, 1961 . 


\section{RÉFÉRENCES}

13. Raff RA, Kaufman TC. Embryos, genes and evolution. New York: Macmillan, 1983.

14. Wolpert I. The evolution of development. Biol J Linn Sor 1990; 39: 109-24.

15. Margulis I. Symbiosis in cell eqolution. New York: Freeman, 1993.

16. Denis H, Lacroix JC: Une interprétation évolutive de la gamétogenèse animale. médecine/sciences 1993; 9: 752-61.

17. Callan HC. Lamplonush chromosomes. Berlin: Springer, 1986.

18. Degnan BM, Iegnan SM, Naganuma T, Morse IDE. The ets multigene family is conserved throughout the Metazoa. Vucleic Acids Res 1993; 21 : 3479-84.

19. Runnegar B. Collagen gene construction and evolution. / Mol Kivol 1985; 22 141-9.

20. Exposito JY, van der Rest M, Garrone $\mathrm{R}$. The complete intron/exon structure of liphydatia mülleri fibrillar collagen suggests a mechanism for the evolution of an ancestral gene module. J Mol livol 1993; 37 254-9.

21. Grell KG. Protozoology. Berlin : Springer, 1973.

22. Denis H, Collenot A. L'origine et l'évolution de la reproduction sexuée. médecine/sciences 1993; 9: 1392-403.

23. Raff EC. Genetics of microtubule systems. J Cell Biol 1984; 99 : 1-10.

24. De Puytorac P, Grain J, Mignot JP. Pré cis de protistologie. Paris: Boubée, 1987.

25. Paintrand $\mathbf{M}$, Moudjou $M$, Delacroix $\mathrm{H}$, Bornens $\mathbf{M}$. Centrosome organization and centriole architecture: their sensitivity to divalent cations. J Struct Biol 1992; 108 : 107-28.

26. Buss LW'. The evolution of individuality Princeton: Princeton University Press, 1987

27. Grassé PP. Protozoaires. Généralités. In : Grassé PP, ed. Traité de zoologie. Tome 1. Premier fascicule. Paris: Masson, 1952 : 37-1.32.

28. Karpov SA, Mylnikov AP. Preliminary observations on the ultrastructure of mitosis in choanoflagellates. Eur J Protistol 1993; $29: 19-23$.

29. Gould SJ. Ontogeny and phylogeny. Cambridge: Harvard University Press, 1977.

30. de Beer (;. Embryos and ancestors. Londres: Oxford University Press, 1958.

31. Gilbert SF. Developmental biology. Sunderland: Sinauer, 1991.

32. Grassé PP, Devillers C. Zoologie. Tome II. Vertétrés. Paris: Masson, 1961.

33. Mignot JP. Les coenobes chez les volvocales: un exemple de passage des unicellulaires aux pluricellulaires. Ann Biol
34. Mazabraud A, Wegnez M, Denis $H$ Origin of several abundant proteins of amphibian oocytes. J Mol livol 1992; 35 : 546-50.

35. Baldauf SI., Palmer JD. Animals and fungi are each other's closest relatives congruent evidence from multiple proteins. Proc Nall Accud Sri USA 1993; 90) : 11558-62.

36. Hynes RO, I ander AI). (Contact and adhesive specificities in the associations, migrations and targeting of cells and axons. Cell 1992; 68: 303-22.

37. Adams JC, Watt FM. Regulation of development and differentiation by the extracellular matrix. Development 1993; 117 : 1183-98.

38. Guadagno TM, Ohtsubo M, Roberts JM, Assoian RK. A link between cyclin A expression and adhesion-dependent cell cycle expression. Science. 1993; 262 : 1472-5.

39. Juliano RI, Haskill S. Signal transduction from the extracellular matrix. I Cell Biol 1993; 120): 577-85.

40. Blobel C.P, Wolfsberg TG, Turck CW Myles I)G, Primakoff P, White IM. A potential fusion peptide and an integrin ligand domain in a protein active in sperm-egg fusion. Vature 1992; 356 . 248-52.

41. Campuzano S, Modolell J. Patterning of the Irosophila nervous system: the achaete-scute gene complex. Trends Genet $1992 ; 8: 202-8$

42. Herbomel P. I'expression du génome du noyau ì l'organisme. Paris: ESTEM, 1993.

43. Laudet V, Hãnni C, Coll J, Catzeflis F, Stéhelin D. Evolution of the nuclear hor mone receptor gene superfamily. $E M B O$ $1992 ; 11: 1003-13$

44. Blenis J. Signal transduction via the MAP kinases: proceed at your own RSK P'roc Natl Acad Sci USA 1993; 90 : 5889-92.

45. Fields $S$. Pheromone response in yeast. Trends Biochem Sic 1990; 15: 27()-3.

46. Heery IDM, Zacharewski T, Pierrat B Gronemeyer $\mathrm{H}$, Chambon $\mathrm{P}$, Losson $\mathrm{R}$. Efficient transactivation by retinoic acid receptors in yeast requires retinoid $X$ receptors. Proc Natl Acad Sci USA 1993; 90 : 4281-5.

47. Hadzi J. The evolution of the Metazoa New York: Pergamon Press, 1963.

48. Raff RA, Kaufman TC. Embryos, genes and evolution. New York: Macmillan, 1983.

49. Pavans de Ceccatty P. Les éponges et la pluricellularité des métazoaires. Ann Bio 1985 ; 24 : 275-87.

50. De Loof A. All animals develop from a blastula: consequences of an undervalued definition for thinking on development. BioEssays 1992; 14: 573-5.

51. Denis H, Lacroix IC. L'origine de la lignée germinale et de la mortalité cellulaire. médecine/sciences $1992 ; 8$ : 695-7()2.
52. Tardent $P$. The differentiation of germ cells in (nidaria. In : Halvorson H(), Monroy $\mathrm{A}$, eds. The origin and evolution of sex. New York: Liss, 1985: 163-97.

53. Nishimiya-Fujisawa C, Sugiyama T Genetic analysis of developmental mechanisms in Hydra XX. Cloning of interstitial stem cells restricted to the sperm differentiation pathway in Hydra magnipapillata. Dev Biol 1993; 157 : 1-9.

54. Granchamp S, Beisson J. Positional control of nuclear differentiation in Paramecium. Dev Biol 1981 ; 81 : 336-41.

55. W'ilson JE, Macdonald PM. Formation of germ cells in Drosophila. Curr Opin Genet Dev 1993 ; 3 : 562-5.

56. Mosquera L, Forristall C, Zhou Y, King ML. A mRNA localized to the vegetal cortex of Xenopus oocytes encodes a protein with a nanos -like zinc finger domain. Development 1993; 117 : 377-86.

57. Rinkevich B, Lauzon RJ, Brown BW'M Weissman IL. Evidence for a programmed life span in a colonial protochordate. Proc Natl Acad Sici USA 1992; 89: 3546-50.

58. Martinez IDE, Levinton IS. Asexual metazoans undergo senescence. Proc Nall Acad Sci USA 1992; 89: 992()-3.

59. Williams C(C. Pleiotropy, natural selec tion and the evolution of senescence. livo lution 1957; 11 : 398-411.

60. Partridge L, Barton NH. Optimality, mutation and the evolution of ageing. Nature 1993; 362: 305-11.

61. Brown WT. Genetics of human aging Re) Biol Res Aging 1985; 2 : 105-14.

62. Faragher RGA, Kill IR, Hunter JAA, Pope FM, Tannock C, Shall S. The gene responsible for W'erner syndrome may be a cell division " counting" gene. Proc Nall Acad Sici USA 1993; 90): 12030-4.

\section{Remerciements}

Les auteurs remercient Janine Beisson, Michel Bornens, Jean-Claude Boucaut, Alain Collenot, Maryvonne Mével-Ninio, Bernard Mignotte, Pierre de Puytorac et Maurice Wegnez pour leurs conseils pendant la rédaction de cet article.

\section{Herinan Denis}

Professeur à l'université Pierre-t-Mariz-Curie. Centre de génétique moléculaire, Cnrs, ave nue de la Terrasse, 91198 Gif-sur-Yvette Cedex, France.

Jean-Pierre Mignot

Professeur à l'université Blaise-Pascal (Clermont-Ferrand-II). Laboratoire de biologie des protistes, 24, avenue des Landais, 63177 Aubière Cedex, France. 


\section{Summary}

The origin of the Metazoa

Alternating phases punctuate the life cycle of protozoans. The vegetative phase consists of a succession of mitoses. The sexual phase converts the vegetative cells into male and female gametes which eventually fuse into a zygote. Protozoan cells successively perform the vegetative program and the sexual program. In metazoans these programs are carried out by different cells. Somatic cells fulfill the vegetative functions, while germ cells fulfill the reproductive functions. The simplest form of embryonic development implies that the zygote gives rise to just two kinds of cells. This article proposes a series of evolutionary transitions to explain the conversion of protozoans into metazoans and the emergence of a rudimentary embryogenesis. Colonies represent plausible intermediates between unicellular and multicellular organisms. They do not undergo a proper embryogenesis as long as they are mere aggregates of identical cells. Embryonic development started when a region of the zygote's cytoplasm acquired factors able to trigger the execution of the sexual program. Among embryonic cells, those receiving the germinal factors took over the reproductive function. The other cells were invested with the vegetative functions.

TIRÉS A PART

H. Denis.

$\mathrm{m} / \mathrm{s} n^{\circ} 5$ vol. 10 , mai 94 